\title{
Image Denoising Method Based on $v$-Support Vector Regression and Noise Detection
}

\author{
Changyou Wang, Zhaolong Gao \\ College of Computer Science and \\ Technology \\ Chongqing University of Posts and \\ Telecommunications, \\ Chongqing, 400065, China \\ wangcy@cqupt.edu.cn
}

\author{
Changyou Wang, Zhaolong Gao \\ Key Laboratory of Industrial Internet \\ of Things \& Networked Control of \\ Ministry of Education, \\ Chongqing University of Posts and \\ Telecommunications \\ Chongqing, 400065, China
}

\author{
Changyou Wang \\ College of Mathematics and Physics, \\ Chongqing University of Posts and \\ Telecommunications \\ Chongqing, 400065, China \\ wangcy@cqupt.edu.cn
}

\begin{abstract}
Aimed at the correlation between noise pixels and neighboring pixels, a new method based on the $V$-support vector regression ( $V$-SVR) is proposed to remove the salt \& pepper noise in corrupted images. The new algorithm first takes a decision whether the pixel under test is noise or not by comparing the block uniformity of the $3 \times 3$ window with one of the entire image, secondly adjusts adaptively the size of filtering window which is used to determine the training set according to the number of noise points in the window, thirdly determines the decision function that is used to predict the gray value of the noise pixels by means of training set, finally removes the noises in terms of the decision function based on $V$-SVR. Experimental results clearly indicate that the proposed method has a better filtering effect than the existing methods such as standard mean filter, standard median filter, adaptive median filter by means of visual quality and quantitative measures.
\end{abstract}

Keywords-Salt \& pepper noise; $V$-support vector regression; Noise Detection; Block uniformity; Image denoising

\section{INTRODUCTION}

Digital image will inevitably be subject to the internal or external noise interference that leads to image quality degradation during image acquisition, storage or transmission in a noisy environment. In such a case, image restoration and enhancement is an essential task and the challenging is to find an efficient technique for noise suppression while preserving the details of image. In impulse noise, also known as salt and pepper noise, noisy pixels take either maximum or minimum value. Thus, it could severely degrade the image quality and cause some loss of information details. To solve the above problems, researchers have proposed various filtering techniques such as the mean filter ${ }^{[1]}$, the median filter ${ }^{[2]}$, the switching median filter ${ }^{[3]}$, and the adaptive weighted median filter ${ }^{[4]}$ etc for removing impulse noise in the past. However, the effect of some of the traditional methods in the removal of image noise and preserving image details can not meet the requirements of the image subsequent processing.

Recently, the support vector regression (SVR) is applied in image denoising ${ }^{[5-8]}$. However, in the previous works, these filters based on SVR apply the SVR operation to each pixel unconditionally without identifying whether a pixel is "corrupted" or "uncorrupted" which would inevitably alter the intensities and remove signal details of those uncorrupted pixels. Therefore, a noise detection process to discriminate the uncorrupted pixels from the corrupted ones prior to applying SVR filtering is highly desirable.

This paper proposes an image denoising method based on $v$-SVR and noise detection in the light of the excellence and shortcoming of traditional median filtering algorithm and the previous SVR filtering algorithm as well as the adaptive weighted median filtering algorithm. The proposed algorithm takes a decision whether the pixel under test is corrupted or not before applying the SVR filter. In order to improve the noise suppression and detail preserving capability of the proposed filter the adaptively determining window length technique and weighted technique are incorporated in the filtering stage.

\section{II. $v$-SUPPORT VECTOR REGRESSION ( $\boldsymbol{v}$-SVR)}

Support vector machine is a machine learning method that is based on the principle of structural risk minimization for small sample. Given training data

$$
\left(X_{1}, y_{1}\right),\left(X_{2}, y_{2}\right), \cdots,\left(X_{l}, y_{l}\right),
$$

where $X_{i}$ represents the input data and $y_{i}$ represents the output data, the traditional linear regression looks for a linear function $\omega^{T} X+b$ that minimizes the mean square error:

$$
\min _{W, b} \sum_{i=1}^{l}\left(y_{i}-\left(\omega^{T} X_{i}+b\right)\right)^{2}
$$

where $\omega$ is the corresponding weight vector for the feature vector $\mathrm{X}$ and $\mathrm{b}$ is the intercept (a constant term). Intuitively, for $1 \mathrm{D}$ case, linear regression is to find a line that crosses all the sample points. A linear regression is not sufficient if the input data is not linearly distributed. Support vector machines use a mapping function $\phi(x)$ to map the data into a higher dimensional space, where a linear function is adequate. Once data is mapped into the high-dimensional space, overfitting can occur. Consequentially, the trained function has poor generalization on test data that usually will never be identical to the training data. To limit overfitting 
thus to make the trained function more applicable on new data, a soft margin and a regularization term are added into the objective function. Support vector regression solves the following optimization problem in order to find a linear function $\omega^{T} X+b$ :

$$
\begin{aligned}
\min _{\omega \in R^{n}, \zeta^{(*)} \in R^{2 l}, \mathcal{E}, b \in R} \tau\left(\omega, \xi^{(*)}, \varepsilon\right)= & \frac{1}{2} \omega^{T} \omega+C \cdot(v \varepsilon \\
& \left.+\frac{1}{l} \sum_{i=1}^{l}\left(\xi_{i}+\xi_{i}^{*}\right)\right)
\end{aligned}
$$

Subject to $\quad\left(\left(\omega \cdot X_{i}\right)+b\right)-y_{i} \leq \varepsilon+\xi_{i}$

$$
\begin{gathered}
y_{i}-\left(\left(\omega \cdot X_{i}\right)+b\right) \leq \varepsilon+\xi_{i}^{*}, \\
\xi_{i} \geq 0, \xi_{i}^{*} \geq 0, \varepsilon \geq 0, i=1,2, \cdots, l,
\end{gathered}
$$

where $\xi_{i}, \xi_{i}^{*}$ are the slack variables, $\xi^{*}=\left(\xi_{1}, \xi_{l}^{*}, \cdots, \xi_{l}, \xi_{l}^{*}\right)^{T}$, and $v, C$ are non-negative parameters.

Using Wolfe duality theorem the primal problem (1)-(4) are converted to the following dual problem

$$
\begin{aligned}
\min _{\alpha^{(*)} \in R^{2 l}} W\left(\alpha^{(*)}\right)= & \frac{1}{2} \sum_{i, j=1}^{l}\left[\left(\alpha_{i}^{*}-\alpha_{i}\right)\left(\alpha_{j}^{*}-\alpha_{j}\right)\right. \\
& \left.K\left(X_{i} \cdot X_{j}\right)\right]-\sum_{i=1}^{l}\left(\alpha_{i}^{*}-\alpha_{i}\right) y_{i},
\end{aligned}
$$

Subject to $\sum_{i=1}^{l}\left(\alpha_{i}-\alpha_{i}^{*}\right)=0, \quad 0 \leq \alpha_{i}, \alpha_{i}^{*} \leq \frac{C}{l}$,

$$
\sum_{i=1}^{l}\left(\alpha_{i}+\alpha_{i}^{*}\right) \leq C \cdot v, C \geq 0, v \geq 0
$$

By solving the optimal solution for the optimization problems

$$
\alpha^{(*)}=\left(\alpha_{1}, \alpha_{1}^{*}, \ldots, \alpha_{l}, \alpha_{l}^{*}\right)^{T}
$$

Thus, the decision function is

$$
f(X)=\sum_{i=1}^{l}\left(\alpha_{i}^{*}-\alpha_{i}\right) K\left(X_{i}, X\right)+b
$$

where $b$ (and $\varepsilon$ ) can be computed by taking into account that equations 2 and 3 become equalities with $\xi^{(*)}=0$ for points with $0<\alpha^{(*)}<C / l$ respectively, due to the $\mathrm{KKT}$ conditions. In this paper, RBF function is chosen as kernel function

$$
K\left(X, X_{i}\right)=\exp \left\{-\frac{\left|X-X_{i}\right|^{2}}{\sigma^{2}}\right\}
$$

Because the noise points have different training set, it must be selected that the appropriate parameters $C, \nu, \sigma$ before the calculation. Commonly used methods are K-fold crossvalidation, grid search and so on. In this paper, we use a grid search method to determine the parameters of $v$-SVR. The method first selects the certain ranges $\left[C_{1}, C_{2}\right],\left[v_{1}, v_{2}\right]$, $\left[\sigma_{1}, \sigma_{2}\right]$ and steps $C_{s}, \nu_{s}, \sigma_{s}$ of parameters $C, v, \sigma$, and then each combination $\left(C_{i}, v_{i}, \sigma_{i}\right)$ is trained respectively. Finally, we take the group parameters whose predict result is the most accurate for the regression prediction of noise point.

\section{DETERMINE OF THE NOISE POINT}

The determining of the noise points of the images, which provides an important basis for the classification of image pixels, is the first step of filtering algorithm and also is the crucial step. In this paper the method proposed in [4] is used to determine noise points. Suppose that the image size is $M \times N$ and the upper left pixel location is at $(1,1)$. First of all, the maximum and minimum gray values of the whole image are found out, and denoted as $\operatorname{Max}\left(P_{M \times N}\right)$ and $\operatorname{Min}\left(P_{M \times N}\right)$. Then the average gray value of the whole image is calculated as follows:

$$
\operatorname{Average}\left(P_{M \times N}\right)=\frac{1}{M \times N} \sum_{i=1}^{M} \sum_{j=1}^{N} f(i, j) .
$$

When determining the noise points, we slide on the image with a $3 \times 3$ sliding window. The gray value of central pixel of this window is $f(i, j)$. The set of the gray values of all the pixels of this window is as follows:

$$
W_{i, j}=\{f(i+k, j+r) \mid k, r=-1,0,1\}
$$

If the gray value of the central pixel of the sliding window $3 \times 3$ is equal to $\operatorname{Max}\left(P_{M \times N}\right)$ or $\operatorname{Min}\left(P_{M \times N}\right)$, they are considered as noise points. Next, we obtain the average value of the non-noise pixels for the sliding window which have been confirmed as Average $\left(W_{i, j}\right)$, and set the number of noise points confirmed for each window as SUM. The formula of calculating Average $\left(W_{i, j}\right)$ is as follows:

$$
\operatorname{Average}\left(W_{i, j}\right)=\frac{1}{9-S U M} \sum_{k=-1}^{1} \sum_{r=-1}^{1} f(i+k, j+r) \text {, }
$$

where $f(i+k, j+r) \neq \operatorname{Max}\left(P_{M \times N}\right)$ and $f(i+k, j+r) \neq \operatorname{Min}\left(P_{M \times N}\right)$.

The noise image can be regarded as a background (original image) superimposed by a noise signal (impulse noise). As long as that the noise signal is higher than the contrast threshold of human visual system (Human Vision System, HVS), the visual system can feel the presence of noise. According to the contrast characteristics of HVS, the threshold value is influenced by the background illuminetion and noise signal frequency. Generally speaking, the lighter the background and the more complex the texture, 
the higher the threshold of the noise intensity HVS can withstand. According to Weber's law, the definition of block uniformity of the sliding window central pixel is given as follows:

$$
d\left(W_{i, j}\right)=\frac{1}{9-\operatorname{sum}} \sum_{\substack{f(i, j) \in W_{i, j} \\ f(i, j) \neq \operatorname{Max}\left(P_{M \times N}\right), \\ \text { and } \neq \operatorname{Min}\left(P_{M \times N}\right)}} \frac{\mid f(i, j) \text {-Average }\left(W_{i, j}\right) \mid}{\operatorname{Average}\left(W_{i, j}\right)}
$$

Here Average $\left(W_{i, j}\right)$ is same as the formula $(13), d\left(W_{i, j}\right)$ denotes the block uniformity of the sliding window $W_{i, j}$ having been filtered noise. That is, the noise threshold that can be felt by HVS exactly in the central pixel $f(i, j)$. The block uniformity of the whole image is as follows:

$$
d\left(P_{M \times N}\right)=\frac{1}{M \times N} \sum_{i=1}^{M} \sum_{j=1}^{N} \frac{\left|f(i, j)-\operatorname{Average}\left(P_{M \times N}\right)\right|}{\operatorname{Average}\left(P_{M \times N}\right)} .
$$

Here Average $\left(P_{M \times N}\right)$ is the average gray value of the whole image in formula (11). According to Weber's law, $d\left(P_{M \times N}\right)$ is the average threshold which the HVS can feel in the whole image.

In summary, the steps of determining noise are as follows:

Step1: If the gray value $f(i, j)$ of the central pixel point $(i, j)$ of the sliding window $3 \times 3$ is equal to $\operatorname{Max}\left(P_{M \times N}\right)$, it will be considered as noise.

Step2: If the gray value $f(i, j)$ of the central pixel point $(i, j)$ of the $3 \times 3$ sliding window is equal to $\operatorname{Min}\left(P_{M \times N}\right)$, it will be considered as noise.

Step3: If the block uniformity of the central pixel of the $3 \times 3$ sliding window is greater than that of the whole image, that is to say $d\left(W_{i, j}\right) \geq d\left(P_{M \times N}\right)$, it will be considered as noise.

According to the rules above, the noise pixel is marked as $N(i, j)=1$ and the non-noise pixel is marked as $N(i, j)=0$.

\section{DETERMINATION OF THE FILTERING WINDOW SIZE}

The noise elimination performance is greatly affected by the size of the filtering window when the standard median filter algorithm is employed. That is, when the filtering window is small, the image detail can be preserved relatively well while noise filtering capability is poor; on the contrary, when the window is large, noise restraint can be enhanced but image will blur. Therefore, in order to combine the advantages of filtering performance in both cases, the idea of determining the filtering window size selfadaptively is proposed in this paper according to the number of noise pixels within the window screen. A $3 \times 3$ window scanning is also used to count the number of noise points. When the central pixel is identified as a noise one, the statistics formula is:

$$
\operatorname{Num}\left(W_{i, j}\right)=\sum_{k=-1}^{1} \sum_{r=-1}^{1} N(i+k, j+r) .
$$

The size of the filtering window $F W_{m, n}$ is determined by $\operatorname{Num}\left(w_{i, j}\right)$ which is related to the number of noise pixels within the $3 \times 3$ window. The calculation approach is:

$$
F W_{i, j}= \begin{cases}3 \times 3, & \operatorname{Num}\left(W_{i, j}\right) \in\{1,2,3\}, \\ 5 \times 5, & \operatorname{Num}\left(W_{i, j}\right) \in\{4,5,6\}, \\ 7 \times 7, & \operatorname{Num}\left(W_{i, j}\right) \in\{7,8,9\}\end{cases}
$$

\section{DENOISING PROCESS USING $\boldsymbol{v}$-SVR FILTER}

After determining the noise and size of filtering window, the image is divided into noise points and non-noise points. For the non-noise points, their gray values are reserved and kept from filtering, but the noise points are removed with a new $v$-SVR filter. The process of removing noise points in corrupted images are as follows:

Step1: Determine the noise points in image according to the method proposed in part III.

Step2: Determine the size of the filtering window using the method introduced in part IV.

Step3: Determine the training set data on the basis of the non-noises in the filtering window.

Step4: Construct decision function ( $v$-SVR filter) to the centre pixel point $X$ within filtering window using the method proposed in part II according to the training set, the corresponding grey value after noise points are filtered as:

$$
f(X)=\sum_{i=1}^{l}\left[\left(\alpha_{i}^{*}-\alpha_{i}\right) K\left(X_{i}, X\right)\right]+b
$$

where $X$ and $X_{i}(i=1,2, \cdots, l)$ indicate respectively the coordinates of the noise point and non-noise points in the corresponding filtering window. Then the output result of the filter is got and used to replace the gray value of a noise point $X$.

\section{EXPERIMENTS AND RESULTS ANALYSIS}

In order to test whether the algorithm proposed in this paper can effectively filter the pulse noise with different densities and reserve the image details, some simulation experiments are carried out on the platform of Matlab7.0 by the standard test image of Lena with size of $512 \times 512$ and 256 gray-scale in Fig1 (a). Firstly, 10\%, 30\%, 50\%, 70\%, and $50 \%$ of the salt and pepper noise was added in order to Figure1 (a). And then image denoising was completed with the algorithm proposed in this paper, the standard $3 \times 3$ and $5 \times 5$ median filter and mean filter, and the new adaptive 
weighted median filter algorithm for elimination of impulsive noise recently reported in [4] respectively. As can be seen in Figure 1, the algorithm proposed in this paper can not only remove the noise but also reserve the detail information of the original image.

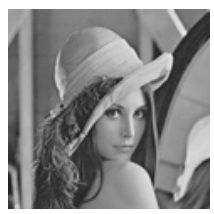

a)

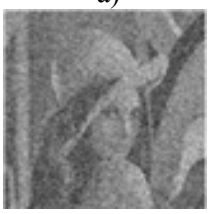

d)

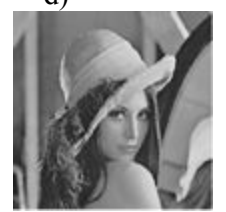

g)

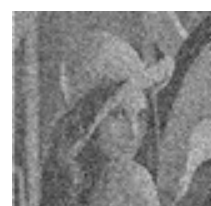

b)

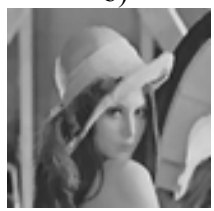

e)

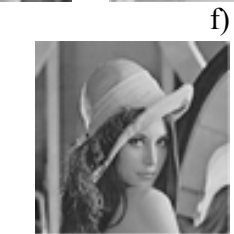

h)

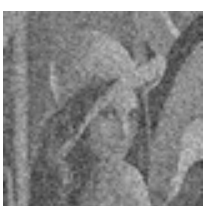

c)

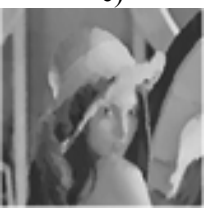

f)
Figure 1. a) original image. b) image with impulsive noise density of $50 \%$. c, d) Filter output of Traditional Mean Filter (TMF) when $3 \times 3$ template and $5 \times 5$ template is used. e, f) Filter output of Traditional Median Filter when $3 \times 3$ template and $5 \times 5$ template is used. g) algorithm processed in [4]. h) Proposed $V_{-S V R}$ filter output.

In order to illustrate the superiority of the new algorithm, the peak signal-to-noise ratio (PSNR) and mean absolute error (MAE) of the output image are used as objective evaluation criteria. PSNR and MAE are defined as following.

$$
\begin{gathered}
P S N R=10 \lg \left(\frac{M \times N \times L^{2}}{\sum_{i, j}[f(i, j)-g(i, j)]^{2}}\right), \\
M A E=\frac{1}{M \times N} \sum_{i, j}|f(i, j)-g(i, j)|,
\end{gathered}
$$

where $\mathrm{L}$ is the maximum gray value of an image. For an image with 256 gray-scales, the value of $\mathrm{L}$ is 255 . Both $\mathrm{M}$ and $\mathrm{N}$ are $512 ; f(m, n)$ is the gray value of experimental image; $g(m, n)$ is the gray value of image having been done de-noising. The evaluation results are in Table 1.

TABLE I. THE PSNR AND MAE OF DIFFERENT ALGORITHMS

\begin{tabular}{|c|c|c|c|c|c|}
\hline \multirow{2}{*}{ Method } & Para- & \multicolumn{4}{|c|}{ Noise density } \\
\cline { 3 - 6 } & meter & $10 \%$ & $30 \%$ & $50 \%$ & $70 \%$ \\
\hline $\begin{array}{c}3 \times 3 \\
\text { Mean Filter }\end{array}$ & $\boldsymbol{P S N R}$ & 24.252 & 19.245 & 16.431 & 14.450 \\
\cline { 2 - 6 } & $\boldsymbol{M A E}$ & 10.785 & 21.229 & 30.172 & 38.511 \\
\hline $\begin{array}{c}5 \times 5 \\
\text { Mean Filter }\end{array}$ & $\boldsymbol{P S N R}$ & 25.521 & 21.205 & 18.243 & 16.063 \\
\cline { 2 - 6 } & $\boldsymbol{M A E}$ & 9.827 & 17.433 & 24.930 & 32.482 \\
\hline $\begin{array}{c}\mathbf{3} \times \mathbf{3} \\
\text { Median Filter }\end{array}$ & $\boldsymbol{P S N R}$ & 32.527 & 29.232 & 24.314 & 17.024 \\
\cline { 2 - 6 } & $\boldsymbol{M A E}$ & 3.030 & 4.143 & 6.385 & 15.055 \\
\hline $\mathbf{5 \times 5}$ & $\boldsymbol{P S N R}$ & 28.420 & 26.006 & 22.764 & 18.356 \\
\hline
\end{tabular}

\begin{tabular}{|c|c|c|c|c|c|}
\hline Median Filter & MAE & 5.079 & 6.695 & 9.981 & 18.015 \\
\hline \multirow{2}{*}{$\begin{array}{c}\text { algorithm } \\
\text { processed in [4] }\end{array}$} & PSNR & 36.246 & 30.779 & 24.584 & 20.044 \\
\cline { 2 - 6 } \multirow{2}{*}{$\boldsymbol{V}$-SVR Filter } & MAE & 0.591 & 2.115 & 5.919 & 8.967 \\
\cline { 2 - 6 } & MAE & 44.232 & 38.308 & 35.058 & 31.942 \\
\hline
\end{tabular}

\section{CONCLUSION}

The adaptive $v$-SVR filter proposed in this paper uses the block uniformity as testing standard to detect the pulse noise on the image. According to the number of noise points detected, the filter window size is adjusted adaptively. Then the training set data is determined on the basis of the nonnoises in the filtering window, and the decision function $(\boldsymbol{V}-$ SVR filter) to the centre pixel point $X$ within filtering window is constructed in accordance with the training set. Finally, the filter value of noise points is calculated according to the decision function. This method, to a large extent, alleviates the conflict between the noise restraint and the image detail reservation so that its ability of filtering noise and reserving image detail is greatly improved. The experimental results show that the new filtering method presented above has better integrated filtering performance than both the standard median filter and the adaptive median filter as well as the new adaptive weighted median filter algorithm recently reported in [4].

\section{ACKNOWLEDGMENT}

This work is supported by Science and Technology Project of Chongqing municipal education committee (Grant no. kJ110501) of China, the Natural Science Foundation Project of CQ CSTC (Grant no. cstc2012jjA20016) of China and the NSFC (Grant no. 40801214) of China.

\section{REFERENCES}

[1] X. M. Zhang, Z. P. Yin, and Y. L. Xiong, "Adaptive switching mean filter using conditional morphological noise detector," Electronics Letters, 2008, vol. 44, no. 6, pp. 406-408.

[2] N.C. Gallagher, G.L. Wise, "A theoretical analysis of properties of the median filters," IEEE Transactions on Acoustics Speech, Signal Processing, 1981, vol. 29, pp. 1136-1141.

[3] Z. Wang, D. Zhang, "Progressive switching median filter for the removal of impulse noise from highly corrupted images," IEEE Transactions On Circuits and Systems, 1999, vol. 46, pp 78-80.

[4] C. Wang, L. LI, F. Yang, and H. Gong, "A new kind of adaptive weighted median filter algorithm," 2010 International Conference on Computer Application and System Modeling (ICCASM 2010). Oct. 2010, Vol. 11, PP. 667-671.

[5] H. Gómez-Moreno, S. Maldonado-Bascón, F. López-Ferreras, P. GilJiménez, "Removal of impulsive noise in images by means of the use of support vector machines," Lecture Notes in Computer Science, 2003, vol.2687, no.2, pp. 536-543.

[6] D. A Marquez, J .L Paredes, W. Garcia-Gabin, "Nonlinear filters based on support vector machines," 2007 IEEE International Conference on Acoustics, Speech and Signal Processing, ICASSP., Dec, 2007, vol.2, pp. 581-584.

[7] D. Li, "Support vector regression based image denoising," Image and Vision Computing, 2009, vol. 27, no. 6, PP. 623-627

[8] F. Yan, N. Ning, "Image de-noising method based on multi-feature combination and weighted support vector machine," Journal of Computer Applications, 2011, vol. 31, no. 8, pp. 2217-2220. 